\title{
From charge transfer type insulator to superconductor
}

\author{
Wei Guo, Rushan Han \\ School of Physics, Peking University, Beijing 100871
}

\begin{abstract}
We propose a microscopic model Hamiltonian to account for impurity doping induced insulator-superconductor transition and the coexistence of antiferromagnetism and superconductivity in the high- $T_{c}$ cuprates. The crossover from non Fermi liquid to Fermi liquid regime characterized by delocalization of $d$ electrons on $\mathrm{Cu}$ sites is discussed.
\end{abstract}

\section{Introduction}

The insulator-superconductor transition driven by impurity doping occurs uniquely in a class of copper oxides with a special magnetic structure. The magnetism of the high- $T_{c}$ cuprate arises from unfilled $d$ shell $\left(3 d^{9}\right)$ of $\mathrm{Cu}^{2+}$ ions in the $\mathrm{CuO}_{2}$ plane, the overlap of the atomic $d$ wave function is weak because of large spacing between $\mathrm{Cu}$ ions $(3.8 \AA)$. The strong correlation between local $\mathrm{Cu}$ moments $(S=1 / 2)$ comes from the indirect interaction mediated by $p$ electrons at neighbor oxygen sites, i.e. the superexchange[1][2]. Even though the superfluid carriers in the high- $T_{c}$ cuprates have the $d$ wave character, however experimental observations confirm that the doped holes actually appear in the $p$ band[3], which is consistent with the superexchange picture since $d$ electrons are localized in the doping range $0<x<$ 0.2 . In this article we show that $d$ wave superconductivity is not originated from hopping $d$ electrons but $p$ electrons. Undoped high- $T_{c}$ cuprates are in fact charge transfer type insulators[4], where $p$ band is above lower $d$ band. $p$ electrons being the mediation for magnetic ordering in the insulating phase became conductive after impurity doping, which causes the partial occupancy of the Wannier $p$ state at a portion of oxygen sites and local $d$-level shift at neighbor $\mathrm{Cu}$ sites. If local $d$-level merge into $p$ band, the hybridization between $p$ and $d$ electrons generates the Kondo exchange interaction between itinerant $p$ electron spins and the local $\mathrm{Cu}$ spin. We show that at such a $\mathrm{Cu}$ site, the local $\mathrm{Cu}$ spin can be a mediation for electron pairing via Kondo exchange. The effective electron-electron interaction originated from the spin coupling is attractive and may yield a bound spin triplet with opposite spins $\chi_{+}=|\uparrow \downarrow\rangle+|\downarrow \uparrow\rangle\left(S=1, S_{z}=0\right)$. The $d$ wave symmetry of paired electrons comes from $l=2$ partial wave channels of Bloch states forced by crystal and spin pair symmetry. Since electron pairs couple to the local spins in the $\mathrm{CuO}_{2}$ plane the effective pair-pair potential is strong and leads to high temperature superfluid transition.

\section{Hamiltonian}

The high- $T_{c}$ cuprates are essentially interacting spin systems. In the low doping limit, its main electronic structure consists of localized $d$ electrons and conductive $p$ electrons. To begin our analysis, we first divide $\mathrm{Cu}$ spins in the $\mathrm{CuO}_{2}$ plane into 
clusters. A spin cluster consists of a $\mathrm{Cu}$ spin at the center of the cluster and four nearest $\mathrm{Cu}$ spins. Interactions between $d$ electron at the central $\mathrm{Cu}$ site and $p$ band electrons can be written in terms

$\hat{H}^{\prime}=\sum_{\vec{k} \sigma} \epsilon_{\vec{k}} \hat{c}_{\vec{k} \sigma}^{\dagger} \hat{c}_{\vec{k} \sigma}+\sum_{\sigma} \epsilon_{d} \hat{d}_{\sigma}^{\dagger} \hat{d}_{\sigma}+\sum_{\vec{k} \sigma}\left(V_{\vec{k}} \hat{d}_{\sigma}^{\dagger} \hat{c}_{\vec{k} \sigma}+V_{\vec{k}}^{*} \hat{c}_{\vec{k} \sigma}^{\dagger} \hat{d}_{\sigma}\right)+U_{d} \hat{n}_{d \uparrow} \hat{n}_{d \downarrow}+\frac{1}{N} \sum_{i} U_{p} \hat{n}_{p \uparrow}^{i} \hat{n}_{p \downarrow}^{i}$

where $\hat{c}_{\vec{k} \sigma}^{\dagger}$ is the creation operator of the Bloch state $\phi_{\vec{k} \sigma}(\vec{r})$ from $p$ band. $\epsilon_{d}$ is the local $d$ level, $V_{\vec{k}}$ is the mixing between $d$ and $p$ electrons. The on-site Coulomb interaction $U_{d}$ and $U_{p}$ at copper and oxygen sites are considered, $\hat{n}_{d \sigma}$ and $\hat{n}_{p \sigma}^{i}$ are occupancy operators for Wannier states $\phi_{d \sigma}$ and $\phi_{p \sigma}^{i}, N$ is the total number of oxygen sites. By using mean field approximation, the term $U_{p} \hat{n}_{p \uparrow}^{i} \hat{n}_{p \downarrow}^{i}$ in Eq.(1) can be written as $U_{p}\left(\hat{n}_{p \uparrow}^{i}\left\langle\hat{n}_{p \downarrow}^{i}\right\rangle+\hat{n}_{p \downarrow}^{i}\left\langle\hat{n}_{p \uparrow}^{i}\right\rangle-\left\langle\hat{n}_{p \uparrow}^{i}\right\rangle\left\langle\hat{n}_{p \downarrow}^{i}\right\rangle\right)$, we can expand $\hat{n}_{p \sigma}^{i}$ in the Bloch representation

$$
\hat{n}_{p \sigma}^{i}=\sum_{\vec{k}} \hat{n}_{\vec{k} \sigma} e^{i \vec{k} \cdot \vec{R}_{i}}
$$

the mean field term can be added to $\epsilon_{\vec{k}}$ in Eq.(1), the normalized $p$ band energy is $\bar{\epsilon}_{\vec{k} \sigma}=\epsilon_{\vec{k}}+U_{0}, \hat{H}^{\prime}$ has the same form as the Anderson impurity model. We can write $U_{0}$ in the average form for the nearest-neighbors

$$
U_{0}=\frac{1}{N_{s}} \sum_{i} U_{p}\left\langle\hat{n}_{p-\sigma}^{i}\right\rangle
$$

$\operatorname{sum} \sum_{i}$ is over the nearest oxygen sites $\left(N_{s}\right)$. For undoped compounds $\left\langle n_{p-\sigma}^{i}\right\rangle=1$, the $p$ band is above local $d$ level for large $U_{0}$ and filled. In this case, the $p$ band and the local $d$ level is separated by a energy gap, the mixing interaction $V_{\vec{k}}=0$. For doped compounds, the doped holes may appear in the nearest oxygen sites of a $\mathrm{Cu}$ site, the partial occupancy of $p$ electron reduces the strength of $U_{0}$ and causes local energy levels shift. If local $d$ level at a $\mathrm{Cu}$ site merge into the $p$ band, the hybridization of $p$ and $d$ electrons $V_{\vec{k}}$ generates Kondo exchange interaction since the Anderson model is equivalent to the Kondo exchange model by a canonical transformation[5]. Therefore, we can derive a $p$ - $d$ exchange term from the mixing interaction in Eq.(1), which is

$$
\hat{H}_{p-d}=-J \mathbf{S}_{\mathbf{0}} \cdot \sigma(0)
$$

where $\sigma(0)$ is the spin density of electrons at $\vec{R}_{0}$. The criterion for localization of a $\mathrm{Cu}$ spin in the metallic environment is given by

$$
\left\langle\hat{n}_{d \sigma}\right\rangle=\frac{1}{2}-\frac{1}{\pi} \tan ^{-1}\left(\frac{\bar{\epsilon}_{d \sigma}-\epsilon_{F}}{\Delta}\right)
$$

$\bar{\epsilon}_{d \sigma}$ is the normalized local $d$ level, $\Delta=\pi \sum_{\vec{k}}\left|V_{\vec{k}}\right|^{2} \delta\left(\epsilon-\epsilon_{F}\right)[6]$. The localization of $d$ electron requires $\epsilon_{F}-\bar{\epsilon}_{d \sigma}>>\Delta, d$ level is far below the Fermi energy in the low doping limit.

The next nearest-neighbors of spin $\mathbf{S}_{\mathbf{0}}$ are four $\mathrm{Cu}$ spins $\mathbf{S}_{\mathbf{j}}$, the indirect interaction between $\mathrm{Cu}$ spins via $p$ - $d$ coupling can be expressed in terms of the superexchange

$$
\hat{H}^{\prime \prime}=\frac{K}{2} \sum_{j} \mathbf{S}_{\mathbf{0}} \cdot \mathbf{S}_{\mathbf{j}}
$$


$\operatorname{sum} \sum_{j}$ is over the nearest $\mathrm{Cu}$ sites of $S_{0}$, the factor $1 / 2$ is taken from the fact that $p$ - $d$ transition occurs at one end of $\mathrm{CuO}$ bond, direct exchange at the other end. In a square lattice we have two superexchange bond instead of four. The superexchange arises from $p$ - $d$ coupling, the coupling constant $K=\rho^{2} J_{d}$, where $J_{d}$ is the coupling constant of direct exchange, $\rho$ the transition integral

$$
\rho=\int d^{3} x \phi_{p}(\mathbf{x}) V \phi_{d}(\mathbf{x})
$$

The superexchange in undoped compounds is strong, $K_{0}=0.1 \mathrm{eV}$. If the doped holes appear in the nearest-neighbor of $S_{0}$, the superexchange as well as $U_{0}$ are suppressed by holes easing the restriction for local $\mathrm{Cu}$ spin flip. On the other hand, the $\mathrm{CuO}$ bond of spin $\mathbf{S}_{\mathbf{j}}$ on the boundary of the cluster is less affected by holes, $U_{0}$ keeps large at site $j$, there is no mixing between $d$ and $p$ electrons at such sites, there is one Kondo scatter per spin cluster on the average, the spin cluster model is different from Kondo lattice model. $\hat{H}^{\prime \prime}$ can be further simplified by considering

$$
\hat{H}^{\prime \prime}=\frac{K}{2} \sum_{j}\left[S_{0}^{z} S_{j}^{z}+\frac{1}{2}\left(S_{0}^{+} S_{j}^{-}+S_{0}^{-} S_{j}^{+}\right)\right]
$$

the last two terms in Eq.(8) flips $S_{j}$, which is a higher energy process since $S_{j}$ is stiff in the robust $\mathrm{CuO}$ bonds. These two terms can be ignored as far as low energy excitation is concerned. In a proper doping range, we have the total Hamiltonian $\hat{H}=\hat{H}^{\prime}+\hat{H}^{\prime \prime}$ by considering interactions from the nearest and the next nearestneighbors of a local $\mathrm{Cu}$ spin in a spin cluster

$$
\hat{H}=\sum_{\vec{k} \sigma} \bar{\epsilon}_{\vec{k} \sigma} \hat{c}_{\vec{k} \sigma}^{\dagger} \hat{c}_{\vec{k} \sigma}+\frac{K}{2} \sum_{j} S_{0} S_{j}-J \mathbf{S}_{\mathbf{0}} \cdot \sigma(0)
$$

where $S_{0}, S_{j}= \pm 1$. The anisotropic Ising-type local spin correlation conforms to the experimental result for spin susceptibility measurement[7]. Magnetic ordering of local spins is a key factor to the pairing mechanism. The existence of $p$ - $d$ exchange interaction is justified by the observed anomalous Hall effect in the normal states of high- $T_{c}$ cuprates, which is a common feature of Kondo-type systems originated from $l$ partial wave of conduction electrons and local moment interaction

$$
\hat{H}_{l} \sim \mathbf{l} \cdot \mathbf{S}_{\mathbf{0}}
$$

The partial wave coupling and its contribution to anomalous Hall effect was calculated by using the Anderson model[8]. This type of interaction is irrelevant to electron pairing, we ignore it in the derivation of Eq.(9).

\section{Spin Paring}

From Eq.(9) we can show the instability of the normal states of $p$ band electrons caused by spin coupling. We consider two electrons interact with the local spin $S_{0}$ via the Kondo exchange, which is

$$
\hat{H}_{\text {int }}=-J \mathbf{S}_{\mathbf{0}} \cdot\left(\sigma_{\mathbf{1}}+\sigma_{\mathbf{2}}\right) \quad(J<0)
$$


we calculate the energy variation to second order

$$
\delta E=\left\langle 0\left|\hat{H}_{\text {int }}\right| 0\right\rangle+\frac{\left\langle 0\left|\hat{H}_{\text {int }}\right| 1\right\rangle\left\langle 1\left|\hat{H}_{\text {int }}\right| 0\right\rangle}{E_{0}-E_{1}}
$$

where $|0\rangle$ is the ground state, $|1\rangle$ the excited state. The local spin flip can be a second order spin interaction effect rather than quantum fluctuation. From Eq.(12) we obtain an effective interaction between two electrons, which is

$$
\hat{H}_{e f}=-\frac{1}{4} \lambda J\left(\sigma_{1}^{-}+\sigma_{2}^{-}\right)\left(\sigma_{1}^{+}+\sigma_{2}^{+}\right),
$$

where $\lambda=J / 2 K, J$ is in order of $10^{-2} \mathrm{eV}[8]$. $\lambda$ can be used as a small parameter in calculation of the bind energy of electron pairs. It can be shown that $\hat{H}_{e f}$ has a bound state solution, which is a spin triplet with opposite spins

$$
\chi_{+}=|\uparrow \downarrow\rangle+|\downarrow \uparrow\rangle
$$

with binding energy

$$
E_{b}=-\frac{1}{2} \lambda J
$$

the energy of pair states $|\uparrow \uparrow\rangle$ and $|\downarrow \downarrow\rangle$ depends on $\left\langle 0\left|S_{i}\right| 0\right\rangle$, which violates the translational invariance in the presence of antiferromagnetic background, therefore parallel spin pairs are unphysical states. It is easy to show that the spin singlet is an excited state. The formation of the unconventional electron pair requires local moment $S=1 / 2$ that forbids non-copper oxides being unconventional superconductors. The intrinsic inhomogenity of electronic states in atomic scale is an indication of local pairing, which is observed by using STM technique[9].

\section{D-Wave Paring}

Local triplet pairing excludes $s$ wave pairing, the wave function of electron pair must have $d$ wave symmetry in restrictions of tetragonal symmetry of crystal lattice. So that Bloch electrons may pair through $l=2$ partial wave channel by taking $m= \pm 1$

$$
\psi\left(x_{1}, x_{2}\right) \sim \sin \theta_{1} \cos \theta_{1} \sin \theta_{2} \cos \theta_{2}\left(\exp i\left(\varphi_{1}-\varphi_{2}\right)-\exp i\left(\varphi_{2}-\varphi_{1}\right)\right)
$$

the partial wave states may give a coherent length in order of $10 \AA$, which has been measured in experiments and is difficult to explain by using terms of atomic Wannier wave function. Since electron motion is limited to the layered $\mathrm{CuO}_{2}$ planes, what we observed is actually its two dimensional projection( with z axis in the plane )

$$
\psi_{\theta} \sim-\cos 2 \theta \quad(-\pi \leq \theta \leq \pi)
$$

where $\theta=\theta_{1}-\theta_{2}$ is the relative coordinate of two electrons, which is invariant in space reflection. However, the three dimensional wave function itself is antisymmetric, so that Eq.(17) is consistent with the spin triplet state. It is difficult to distinguish a singlet and a triplet state with opposite spins in experiments, a convincing experimental evidence for identifying the spin state of the electron pair in high- $T_{c}$ cuparates is still lack. The magnetic pair with $S=1$ breaks time-reversal symmetry when it interacts with external probes. Experimental observation of time-reversal symmetry breaking effect in the high- $T_{c}$ cuprates has been reported by a research group by using circularly polarized photon technique[10], which we consider is an indication for the existence of magnetic pair state. 


\section{$5 \quad$ Mean Field Theory}

By using mean field theory to calculate the superfluid transition temperature we obtained a BCS-like expression

$$
k_{B} T_{c}=\omega_{0} \exp \left(\frac{1}{N(0) V}\right)
$$

where $\omega_{0}=\left|E_{b}\right|$, the character energy scale for magnetic coupling, $N(0)$ is the density of states at the Fermi surface[11]. Since electron pairs couples to the whole $\mathrm{CuO}_{2}$ plane, the effective pair potential $V$ is strong. Strongly coupled pair-pair interaction may give rise to the pseudogap in excitation spectrum and linear temperature dependence of resistivity in the normal states[12]. To explain the electronic phase diagram, we calculate the binding energy $E_{b}$ to fourth order in $J$ and take the strong coupling limit $N(0) V>>1$, we obtain an expression for $T_{c}$

$$
k_{B} T_{c}=\frac{1}{2} \lambda J\left(1-\frac{1}{2} \lambda^{2}\right)
$$

where $T_{c}$ increases with increasing hole concentration $x$ via $\lambda$ until a maximum $T_{c}$ is reached

$$
\lambda_{m}^{2}=\frac{2}{3}
$$

Kondo coupling constant $J$ sets a natural limit on the maximum $T_{c}$. By estimating $J$ up to $50 \mathrm{meV}$, the upper limit of $T_{c}$ is about $150 \mathrm{~K}$. The universal expression for $T_{c}$ near optimum doping can be derived from Eq.(19), which is

$$
\frac{T_{c}}{T_{m}}=1-\kappa\left(x-x_{m}\right)^{2}
$$

with

$$
\kappa=\left(\frac{2 K_{0}}{J}\right)^{2}
$$

A analysis of experimental data for $\mathrm{La}_{2-x} \mathrm{Sr}_{x} \mathrm{CuO}_{4}$ gives $\kappa=92.4$, where we have taken $J=26 \mathrm{meV}, K_{0}=0.125 \mathrm{eV}[12]$. Theoretical value of $\kappa$ is close to the empirical value $82.6[13] . \kappa$ and $x_{m}$ are not independent parameters in spin pairing theory, we can show the relation between them. $p$ electron wave function $\psi_{p}$ can be written in terms

$$
\psi_{p}=\mu|p\rangle+\nu|0\rangle
$$

where $|p\rangle$ is the occupied state, $|0\rangle$ is the empty state, $\mu^{2}$ and $\nu^{2}$ are probabilities for $p$ electron and hole occupancy satisfying $\mu^{2}+\nu^{2}=1$. The strength of the superexchange is reduced by holes in a doped high- $T_{c}$ cuprate $K=K_{0}\left(1-\nu^{2}\right)$. At optimum doping $\nu_{m}$ is determined by

$$
\lambda_{m}=\frac{J}{2 K_{0}\left(1-\nu_{m}^{2}\right)}
$$

By taking empirical value $\kappa=82.6$, we obtain $\nu_{m}^{2}=0.85$ from Eq.(24). If hole appears at two oxygen sites in a spin cluster, the optimum impurity concentration is therefore $x_{m}=2 \times 0.85 / 10=0.17$. The positive charge of holes balances the Coulomb repulsion of electrons pair which has a short coherent length. It is easy to 
show from Eq.(15) that a portion of $p$ electron becomes superfluid carriers, in the low doping limit the superfluid density $n_{s}$ increases linearly with the doped hole concentration $x$, thus we have

$$
T_{c} \propto n_{s} \propto x
$$

The superexchange vanishes at a doping point where there are two holes with occupancy $\nu^{2}=1$ residing in a spin cluster, the average hole occupancy in the oxygen sites is $1 / 2$. The condition for charge balance at the central spin site requires $\left\langle\hat{n}_{d, \sigma}\right\rangle=1 / 2$. From Eq.(5), the local level shift caused by impurity doping satisfies $\bar{\epsilon}_{d}-\epsilon_{F}=0, d$ electron is delocalized at the doping concentration $x=0.2$. The superconducting phase also vanishes at this doping point. The delocalization of electrons also causes a crossover from non Fermi liquid to Fermi liquid, since non Fermi liquid behavior actually arises from electron-local-moment interaction.

\section{Summary}

In summary, we use a microscopic model Hamiltonian to describe insulator- superconductor transition, the coexistence of antiferromagnetism and superconductivity in high- $T_{c}$ cuprates. By increasing impurity concentration we have the following effects: i) metal-insulator transition, ii) Kondo scattering at a portion of random $\mathrm{Cu}$ sites, iii) Ising-like short range magnetic order, iv) spin pairing and the superconducting transition, v) delocalization of $d$ electron and crossover from non Fermi liquid to Fermi liquid regime. We have shown that $d$ wave superconductivity is not originated from hopping $d$ electrons but $d$ channels of Bloch electrons from $p$ band forced by spin pairing. High temperature superfluid transition is not due to the strong charge coupling between correlated electrons, but the weak electronic correlation established in a strongly correlated magnetic background. An external probe ( circularly polarized photons for example ) acting on a magnetic electron pair may break time-reversal symmetry, which is the core prediction of spin pairing theory, yet to be confirmed by experimentalists. As we have shown, the concept of spin pairing is capable of achieving a thorough comprehension of the origin of high- $T_{c}$ superconductivity.

\section{References}

1. Kramer H A, 1934 Physica 1182

2. Anderson P W, 1950 Phys. Rev. 79350

3. Romberg H, Alexander M, Nucker N, Adelmann, Fink J, 1990 Phys. Rev. B 42 8768

4. Uchida S, Ido T, Takagi H, Arima T, Tokura Y, Tajima S, Phys. Rev. B 43 7942

5. Schrieffer J R, Wolff P A, 1966 Phys. Rev. 149491

6. Anderson P W, 1961 Phys. Rev. 12441

7. Lavrov A N, Ando Y, Komiya S and Tsukada I, 2001 Phys. Rev. Lett. 87 17007

8. Levy P M, Guo W and Cox D L, 1988 J. Appl. Phys. 633896

9. Pan S-H, O'Neal J P, Badzey R L, Chamon C, Ding H, Engeibrecht, Wang Z, 
Eisaki H, Uchida S, Gupta A K, Ng K-W, Hudson E W, Lang K M and Davis J C, 2001 Nature 413282

10. Kaminski A, Rosenkranz S, Fretwell H M, Campuzano J C, Li Z, Raffy H, Cullen W G, You H, Olson C G, Varma C M and Hochst H, 2002 Nature 416610 11. Guo W and Han R-S, 2001 Physica C 364-365 79

12. Guo W and Han R-S, 2002 Chin. Phys. Lett. 191687

13. Tallon J L, Cooper J R, I.P.N. de-Silva P S, Williams G V M and Loram J W, 1995 Phys. Rev. Lett. 75 4114; Tallon J L, Bernhard C, Shabed H, Hatterman R L and Jorgenson J D, 1995 Phys. Rev. B 5112911 